\title{
Self mode locked OPSL: role of high- order modes in starting self-mode- locked operation (withdrawal notice)
}

H. Liang, Y. Lee, J. Tung, K. Su, Y. Chen, et al.

H. C. Liang, Y. C. Lee, J. C. Tung, K. W. Su, Y. F. Chen, K. F. Huang, "Self mode locked OPSL: role of high-order modes in starting self-mode-locked operation (withdrawal notice)," Proc. SPIE 8606, Vertical External Cavity Surface Emitting Lasers (VECSELs) III, 86060M (18 February 2013); doi: 10.1117/12.2006159

SPIE. Event: SPIE LASE, 2013, San Francisco, California, United States 


\section{Self mode locked OPSL: role of high-order modes in starting self-mode-locked operation (withdrawal notice)}

Proc. SPIE 8606, 86060M (2013); http://dx.doi.org/10.1117/12.2006159

Online Publication Date: 18 February 2013

Retracted from Publication: 23 April 2013

Conference Date: 3-5 February 2013

Conference Location: San Francisco, California, USA

Conference Title: Vertical External Cavity Surface Emitting Lasers (VECSELs) III

Conference Chair: Jennifer E. Hastie

H. C. Liang, Y. C. Lee, J. C. Tung, K. W. Su, Y. F. Chen, and K.F. Hunag

National Chiao Tung University (China)

This paper was inadvertently published and has been withdrawn by the publisher.

Vertical External Cavity Surface Emitting Lasers (VECSELs) III, edited by Jennifer E. Hastie, Proc. of SPIE Vol. 8606, 86060M - () 2013 SPIE

CCC code: $0277-786 \mathrm{X} / 13 / \$ 18 \cdot$ doi: $10.1117 / 12.2006159$ 